A revised manuscript submitted to

IEEE Transactions on Antennas and Propagation

as a Communication

\title{
Investigating the Impact of Smart Antennas on SAR ${ }^{1}$
}

\author{
Kwok-Chi Chim, Kenneth C. L. Chan and Ross D. Murch ${ }^{2}$ (Senior Member, IEEE) \\ Department of Electrical \& Electronic Engineering \\ The Hong Kong University of Science and Technology \\ Clear Water Bay, Kowloon, Hong Kong.
}

\begin{abstract}
In this communication we investigate the impact of smart antennas on SAR at $1.9 \mathrm{GHz}$. We emulate the operation of the smart antennas by utilizing a mobile telephone prototype configured with two PIFA antennas and applying carriers with different signal phases to them. The SAR of this prototype is simulated using FDTD and is also experimentally determined using a MapSAR system. It is found that the operation of smart antennas on SAR has a significant impact, with SAR changing by over $50 \%$ for various signal phases. This leads to the conclusion that if smart antennas are to be utilized in handsets careful investigation of their impact on SAR is needed.
\end{abstract}

\footnotetext{
${ }^{1}$ Supported by Hong Kong Research Grants Council HKUST 6048/00E.

${ }^{2}$ Corresponding Author, Phone: (+852) 2358 7044. Fax: (+852) 23581485.

Email: eermurch@ee.ust.hk.
} 


\section{Introduction}

Smart antennas and multiple input multiple output (MIMO) antenna systems are seen as one of the potential technologies for enhancing performance and capacity of future wireless communication systems [1-5]. They are based on utilizing multiple antennas for both transmission and reception of signals and can allow performance and capacity enhancement without the need for additional power or spectrum [1-3].

Wireless communication devices have also become associated with possible health effects surrounding the exposure of humans to electromagnetic radiation [6,7]. One of the most widely used parameters for the evaluation of exposure is the Specific Absorption Rate (SAR). Having low SAR not only reduces electromagnetic exposure but also increases the efficiency of the antenna systems and therefore the battery life of the communication system. Research has focused on measuring, modeling and determining the effects of SAR on humans [6-9]. Efforts have also focused on ways to design antennas to reduce SAR exposure [9-11]. Reduction of SAR can be achieved by minimizing the near field impinging on the human head [9]. The effect of coupling between the chassis and antenna [11] as well as antenna directivity issues for systems operating at Ka bands [10] have also been investigated. Furthermore, nearly all newly proposed handset antennas now consider SAR an important design specification that they must meet. Few papers however consider the effect of SAR when smart antennas are introduced at the handset $[13,14]$.

The objective of this work is to evaluate the effect of smart antennas on SAR at 1.9GHz. We utilize a handset prototype with two planar inverted F antennas (PIFA) to form the smart antenna system. When the smart antennas are used for uplink 
transmissions we emulate the antenna weights by applying different signal phases (while keeping the total transmit power constant) to the PIFA antenna feeds and measure (and simulate) the resulting SAR. Alternatively if the smart antennas were only used for reception (downlink communication), the antenna weights would be freely available for reducing SAR when uplink transmissions occur. In both cases knowledge of the variation of SAR with the weights allows us to determine the impact of smart antennas on SAR. It may also lead us to techniques for minimizing SAR.

\section{Configuration}

The equivalent system model we assume for the smart antennas is shown in figure 1. The wireless communication signal to be transmitted is passed through a splitter and then multiplied by antenna weights before being radiated by the PIFA antennas on the handset prototype. In practice the antenna weights maybe determined by some appropriate uplink algorithm (such as those based on MMSE [2,5]) or set freely if the smart antennas are used for downlink communications only.

The geometry of the PIFAs and printed circuit board (PCB) layout for our prototype are shown in Figure 2. The dimension of the PCB is $l=110 \mathrm{~mm}, w=50 \mathrm{~mm}$ while the size of the PIFA's are $P_{L}=34 \mathrm{~mm}, P_{w}=10 \mathrm{~mm}$ with heights of $h=6 \mathrm{~mm}$ and PIFA separation, $d=20 \mathrm{~mm}$. The corresponding S-parameters for the prototype is shown in Figure 3 where it can be observed that both antennas achieve $S_{11}$ and $S_{22}$ matches of less than $-10 \mathrm{~dB}$ at around $1.9 \mathrm{GHz}$. The $\mathrm{S}_{21}$ coupling between the antennas is less than $-9 \mathrm{~dB}$. Although mutual coupling of $-9 \mathrm{~dB}$ is not insignificant it is generally accepted that envelope signal correlations between the antennas in realistic mobile environments only needs to be less than $0.5[2,4,5]$. Using the bound $\rho_{e}<25\left|S_{12}\right|^{2}$ (which is valid if the 
magnitude of the S-parameters satisfy the condition $\left|S_{11}\right|+\left|S_{22}\right|+\left|S_{11}\left\|S_{22}|+| S_{12}\right\| S_{21}\right|<0.25$ and is also satisfied in our experiment) we can show that $\rho_{e}<0.39$ indicating that sufficient diversity would be achieved $[2,4,5]$.

The head phantom we utilize is a spherical head phantom and is shown in figure 4 . The model is the same as that utilized in the mapSAR equipment we employ for the measurements (see next section). Although it is a simplified head model, it allows the mapSAR equipment to quickly obtain SAR measurements that are sufficiently accurate for the design phase of mobile handset antennas (further information is currently available at http://www.indexsar.com/benchtop.htm). The phantom is constructed from a glass flask filled with liquid head material and the inner diameter of the glass flask is $198 \mathrm{~mm}$ and the thickness of glass is $4 \mathrm{~mm}$. The density for the liquid head material inside the flask is $1000 \mathrm{~kg} / \mathrm{m}^{3}$ and the conductivity and relative dielectric constant are 1.42 and 39.9 respectively at $1.9 \mathrm{GHz}$. The density, conductivity and relative dielectric constant for glass flask are $1000 \mathrm{~kg} / \mathrm{m}^{3}, 0.0018$ and 4.6 respectively.

\section{Results}

Experimental SAR measurements for the head phantom were performed using the MapSAR system produced by indexSAR (further information is currently available at http://www.indexsar.com/benchtop.htm). The signal for the SAR measurement was formed by passing a 1.9GHz carrier generated by an HP8753D signal generator through a power splitter to form two identical signals but with different phases. Specifically we used the Mini-Circuits ZFSC-2-2500 power splitter for producing 0 degree phase shifts, the Mini-Circuits ZAPDJ-2 power splitter for producing 180 degree shifts and the Narda 
4333 splitter for the 90 degree and 270 phase shifts. All insertion losses were calibrated out of the measurement results. Each signal was then connected to one of the PIFA feeds, where it was noted that the feed of one PIFA was slightly longer than the other and this corresponded to an additional 20 degree phase shift which is incorporated in the experimental results. A power meter was utilized to measure the actual power input to the antennas so that impact of return loss from the mutual coupling was removed. Calibration of the experimental system was performed by using a single dipole calibration antenna consisting of a standard dipole and balun operating at $1.9 \mathrm{GHz}$ and located at $S=4 \mathrm{~mm}$ from the glass surface and centered along the vertical coordinate axis in figure $4 \mathrm{~b}$.

Simulations of SAR were also performed to allow further verification of the trends. These were performed using the FDTD software XFDTD using the same prototype and head phantom as described in section III. The two feed signals required in our simulations were formed by using two separate sources with a specified phase difference. The calibration procedure used in the experimental system was repeated by simulation to calibrate the FDTD simulation results to the MapSAR system.

All our SAR results are specified as maximum $1 \mathrm{~g}$ SAR and are normalized to $1 \mathrm{~W}$ radiated power for the simulations and in the experimental setup it is normalized to $1 \mathrm{~W}$ actual power input to the antennas where any return loss from the mutual coupling has been removed.

The first set of results we provide are shown in figure 5 when our prototype handest (see figure 4) is located at $S=4 \mathrm{~mm}, H=80 \mathrm{~mm}$ and $\mathrm{V}=20 \mathrm{~mm}$. Both simulation and experimental results (the experimental phase shifter described above only produced phase shifts of $0,90,180$ and 270 degrees and therefore only 4 experimental points are shown) 
are provided when both feeds are operating. Although there is some discrepancy between the experimental and simulation results both show that SAR varies by more than $50 \%$ depending on the phase angle between the weights. When only one feed is connected the measurement $\mathrm{SAR}$ is around $5 \mathrm{~mW} / \mathrm{g}$ while the minimum and maximum SAR with both feeds connected is between $2 \mathrm{~mW} / \mathrm{g}$ and $7 \mathrm{~mW} / \mathrm{g}$ respectively. This demonstrates that the use of smart antennas for uplink transmission could have a significant impact on SAR.

To determine if the SAR is sensitive to variation in the position of the prototype we have provided in figure 6 and 7 results when the handset is moved. In figure 6 we provide experimental and simulation results for when $\mathrm{H}$ is increased by $6 \mathrm{~mm}$. Very little change in the results occurs indicating that SAR may not be very sensitive to position in this case. In addition in figure 7 we provide simulation results for more positions of the prototype when $\mathrm{H}$ and $\mathrm{V}$ are both changed be other amounts. These again show that SAR is not particularly sensitive to handset position.

In figure 8 we provide simulation results for three frequencies between 1.8-2.0G and these again show that no significant change in the SAR results occur.

\section{Discussion}

A key question arising from our results is why the SAR varies with the phase difference between the feeds in the way observed. Typically the location of the maximum SAR of a particular head and handset configuration is usually associated with a region near the surface of the head where the handset is located. In our analysis we believe that the fields in this region can be changed by the use of the smart antennas so that the SAR can be altered. In our example we can understand this effect by considering possible even and 
odd mode excitation of the two PIFA antennas. When the phase difference between the feeds is zero we can think of it as an approximate even mode and the fields then must be approximately symmetrical about a plane vertically centered between both PIFA's and no electric feed lines will pass through that plane. Therefore the electric field lines from each PIFA will be diverted away from each other and the SAR in the region near the surface of the head close to the center of both PIFAs will most likely be reduced. On the other hand the odd mode SAR, when the feeds are 180 degrees out of phase will have electric field lines that "connect" both PIFAs together, concentrating the field strength in the center between both PIFAs. The SAR at the surface of the head in the region near the center of the PIFAs will most likely be increased. Both these trends are born out by our results where a minimum is approximately at zero phase and a maximum in SAR approximately at 180 degrees. Other phase shifts for the feeds can be formed by appropriate combinations of the odd and even modes. In particular the peak SAR can be "scanned" from left right in the region near the surface of the head in close proximtry to the handset by appropriately shifting the phase. The 0 degree phase corresponds exactly to when the peak is split between both the right and left sides. This effect may be evident from the results in figure 6 and 7 where we can see that slight variations in the feed phase difference are required to maximise the SAR as we move the handset back and forth along the head by changing $\mathrm{H}$.

\section{Conclusion}

We show that the operation of smart antennas on SAR will have a significant impact when used in uplink transmissions. In particular signal or feed weights cause 
significant variations in SAR depending on the exact phase difference between the feed weights. It can therefore be concluded that the use of smart antennas at the handset will need to be carefully considered if specified SAR levels are important to meet. The results also indicate that additional research is needed in several areas including the effect of weight magnitude, antenna mutual coupling and the size of practical implementations.

\section{Acknowledgement}

We would like to express our thanks to Jason Squires, and the team at Integra Antennas and also the support of the Hong Kong Research Grant Council with grant HKUST 6048/00E.

\section{References}

[1] A. Lozano, F. R. Farrokhi and R. A. Valenzuela, "Lifting the Limits on High-Speed Wireless Data Access Using Antenna Arrays," IEEE Commun. Mag., Vol. 39, pp. 156-162, Sept. 2001

[2] J.H. Winters, "Smart Antennas for Wireless Systems," IEEE Personal Commun. Mag., Vol 5, No. 1, pp. 23-27, Feb. 1998.

[3] RD Murch and KB Letaief, Antenna Systems for Broadband Wireless Access, IEEE Communications Magazine, Vol 40, No 4, pp76-83, April 2002

[4] C. K. Ko and R. D. Murch, "Compact integrated diversity antenna," IEEE Trans. Antenna Propagat., vol. 49, no. 6, pp. 954-960, June 2001.

[5] RG Vaughan and J Bach Andersen, "Antenna Diversity in Mobile Communications," IEEE Trans. Vehicular Technology, Vol. 36, No. 4, pp. 149-172, Nov. 1987.

[6] A. Rosen, A. Vander Vorst and Y. Kotsuka (Eds), "Special Issue pm Medical Applications and Biological Effects of RF/Microwaves", IEEE Trans on Microwave Theory and Techniques, Vol 48, No 11, Part II, November 2000

[7] A. Rosen and A.V. Vorst. (Eds), "Special Issue on Medical Application and Biological Effects of RF/Microwaves" IEEE Trans on Microwave Theory and Techniques, Vol 44, No 10, Part II, October 1996

[8] J.C. Lin, Specific absorption rates (SARs) induced in head tissues by microwave radiation from cell phones, IEEE Antennas and Propagation Magazine, Vol 42 No 5, Oct 2000 pp138 -139

[9] N. Kuster and Q. Balzano, "Energy absorption mechanism by biological bodies in the near field of dipole antennas above 300MHz," IEEE Trans. Vehicular Tech., vol. 41, no. 1, pp. 17-23, February 1992.

[10] W Kang, Y. Kim, and Rahmat-Samii, Handset antennas and humans at Ka-band: the importance of directional antennas IEEE Transactions on Antennas and Propagation,, Vol 46 No 6, Jun 1998, pp $949-950$

[11] P Vainikainen, J. Ollikainen, O Kivekas, K Kelander, Resonator-based analysis of the combination of mobile handset antenna and chassis, IEEE Transactions on Antennas and Propagation, Vol 50, No 10 , Oct 2002, pp1433 -1444 
[12] K. Ogawa, "An analysis of the performance of a handset diversity antenna influenced by head, hand and shoulder effects at 900MHz: Part I - Effective Gain Characteristics," IEEE Trans. Vehicular Tech., vol. 50, no. 3, pp. 830-844, May 2001.

[13] MA. Mangoud, RA. Abd-Alhameed, NJ. McEwan, PS Excell, EA Abdulmula, SAR reduction for handset with two-element phased array antenna computed using hybrid MoM/FDTD technique Electronics Letters, Vol 35 No. 20, 30 Sep 1999, pp 1693 -1694

[14] Kwok-Chi Chi and RD Murch Investigating the effect of smart antenna on SAR, IEEE International Symposium Antennas and Propagation Society, 2002, pp 432 -435

[15] C. R. Rowell and R. D. Murch, "A capacitively loaded PIFA for compact mobile telephone handsets," IEEE trans. Antenna Propagation., vol. 45, pp. 837-842, May 1997.

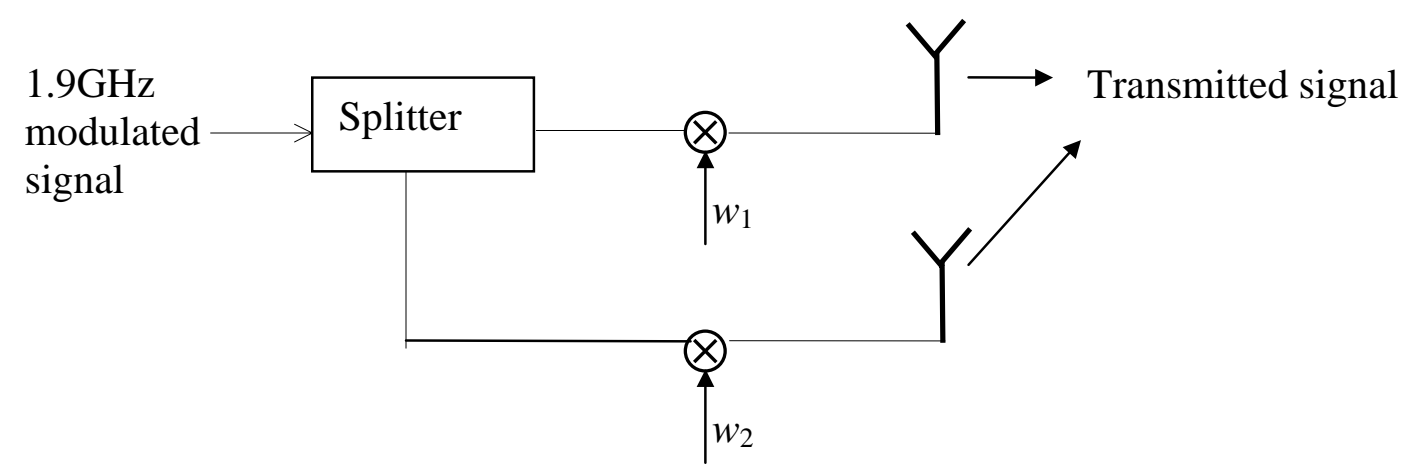

Figure 1: Uplink system model of the smart antennas at the handset.

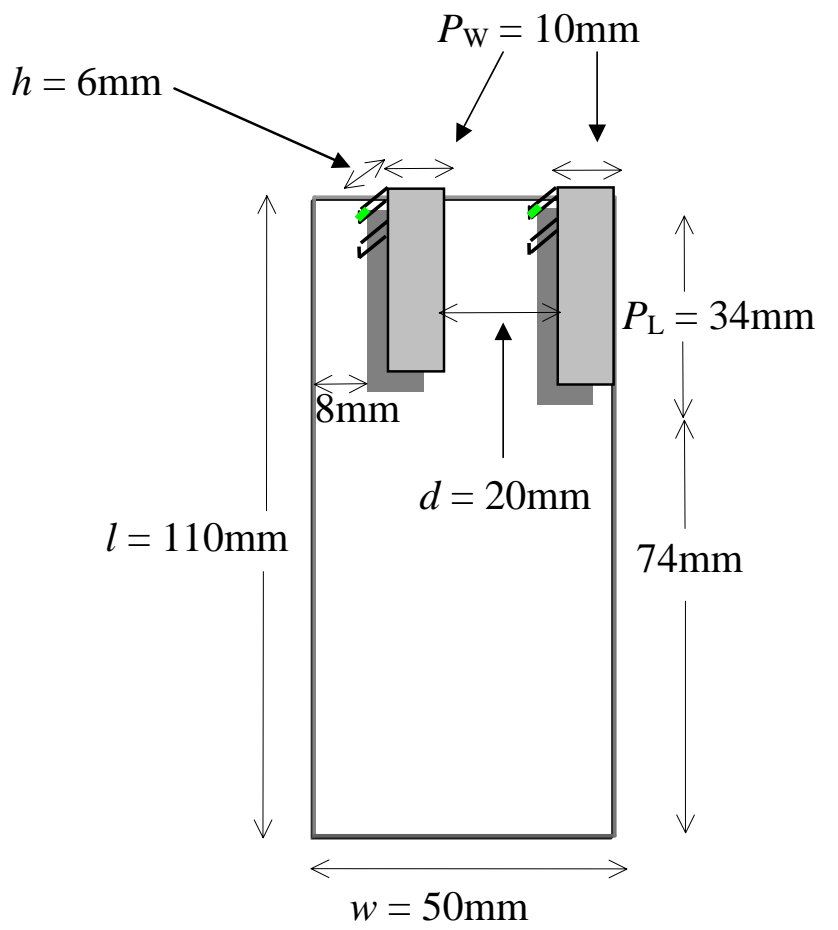

Figure 2. Geometry of the PIFAs and printed circuit board mounting 


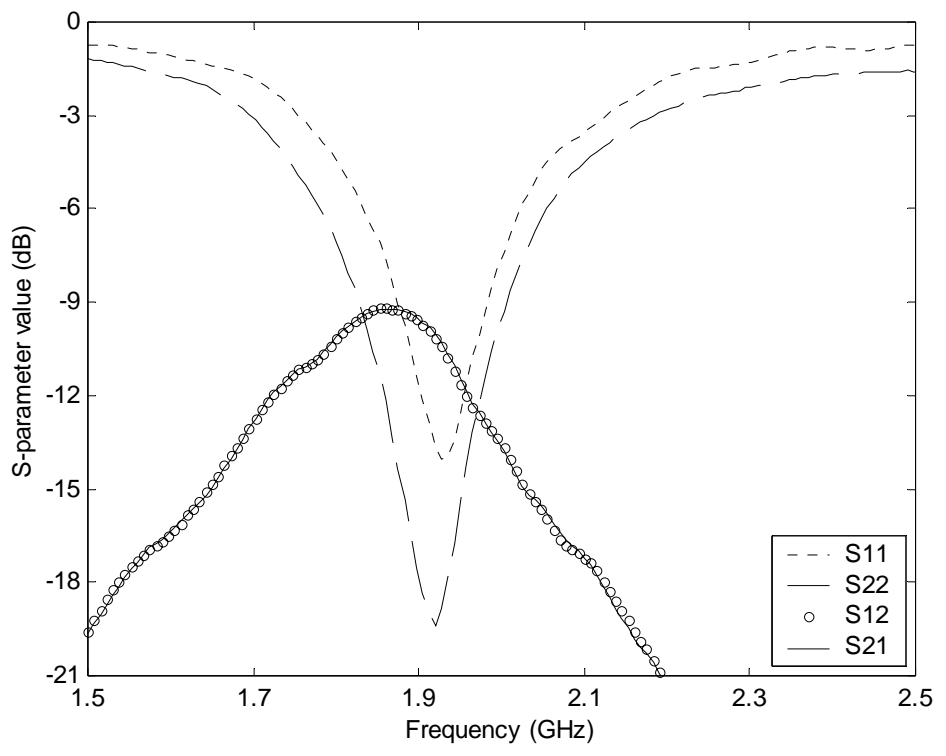

Figure 3: Experimental S-parameter results of our prototype

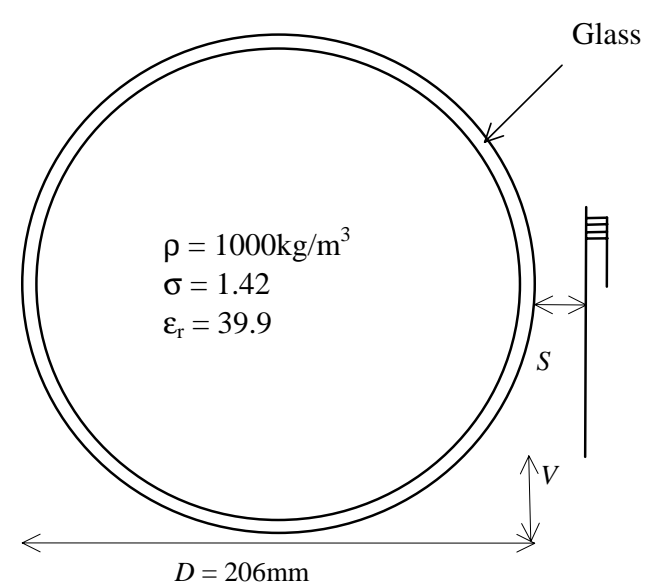

a) Front View

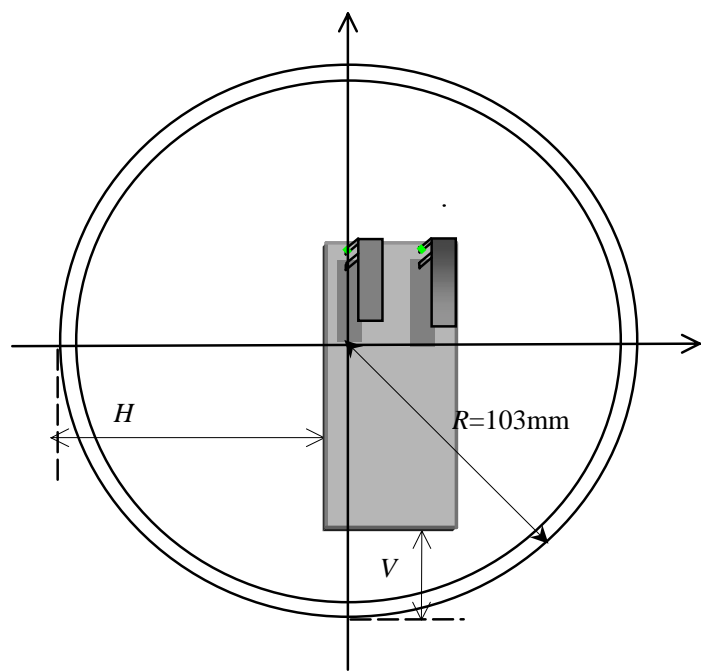

b) Side View

Figure 4: Head model and handset configuration. 


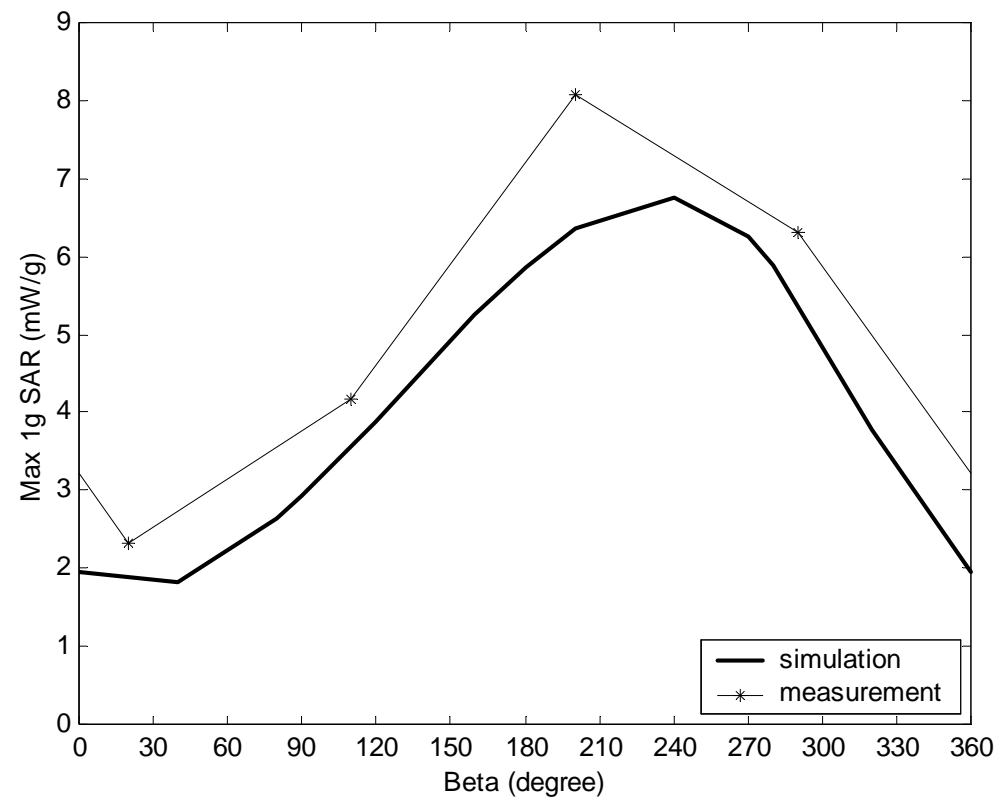

Figure 5. Maximum 1g SAR verses weight phase (Beta) for the handset located at $S=$ $4 \mathrm{~mm}, H=80 \mathrm{~mm}$ and $\mathrm{V}=20 \mathrm{~mm}$ at $1.9 \mathrm{GHz}$.

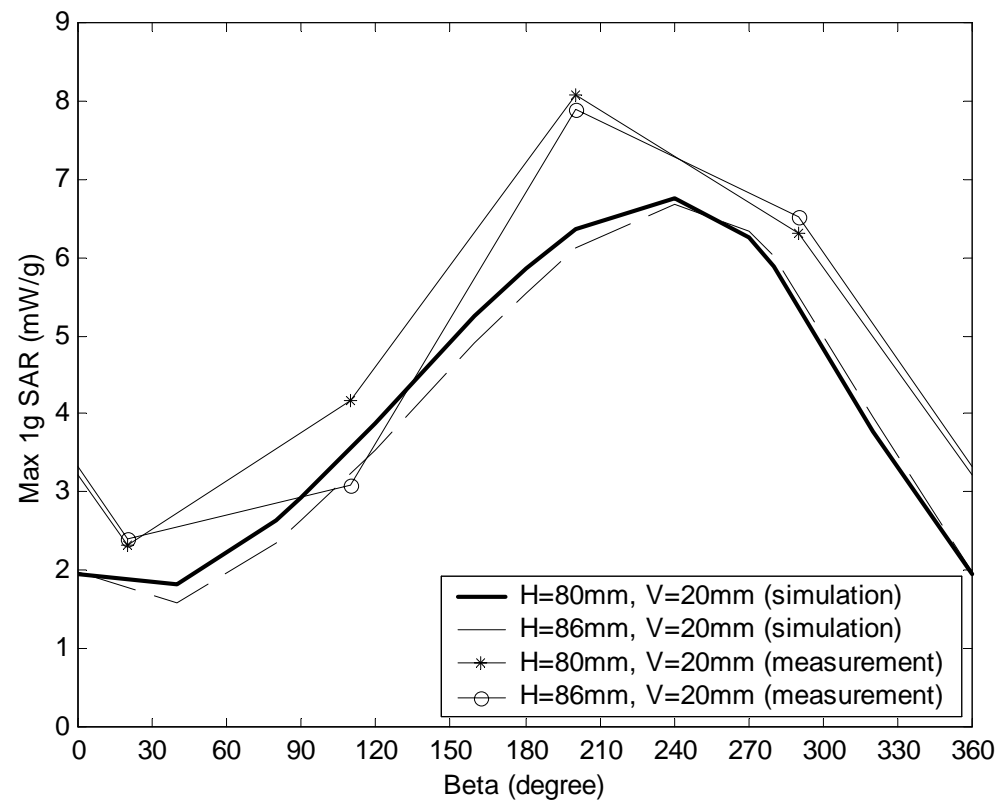

Figure 6. Maximum 1g SAR verses weight phase (Beta) for two handset positions. 


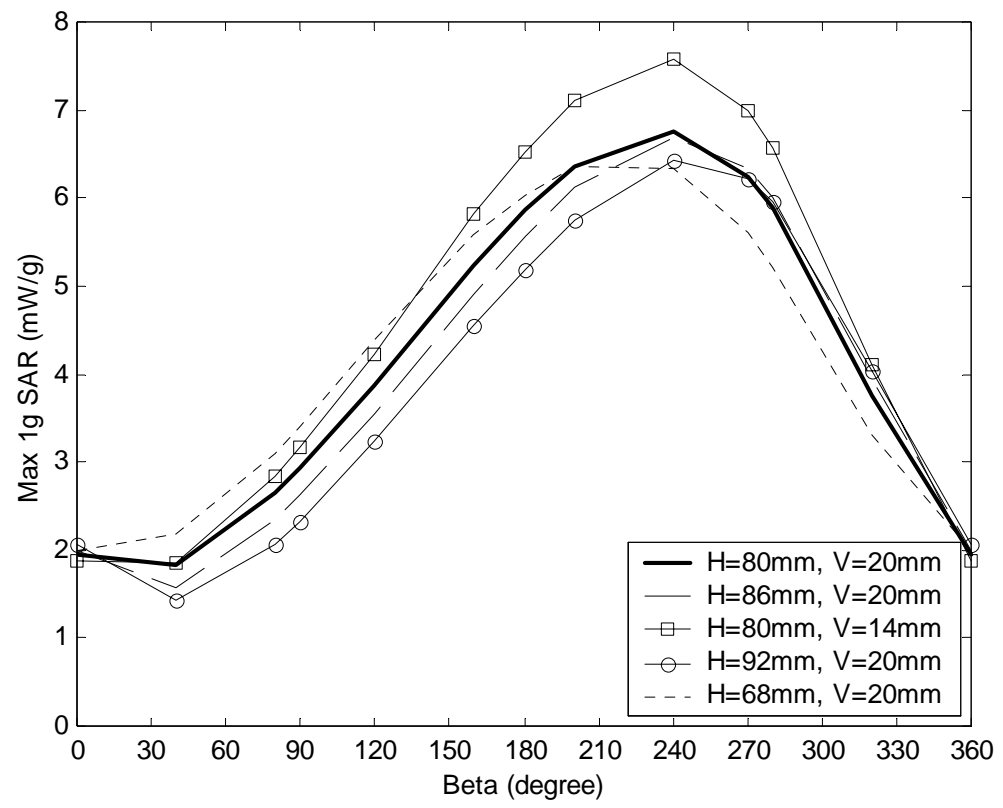

Figure 7. Simulation of maximum $1 \mathrm{~g}$ SAR verses weight phase (Beta) for five handset positions.

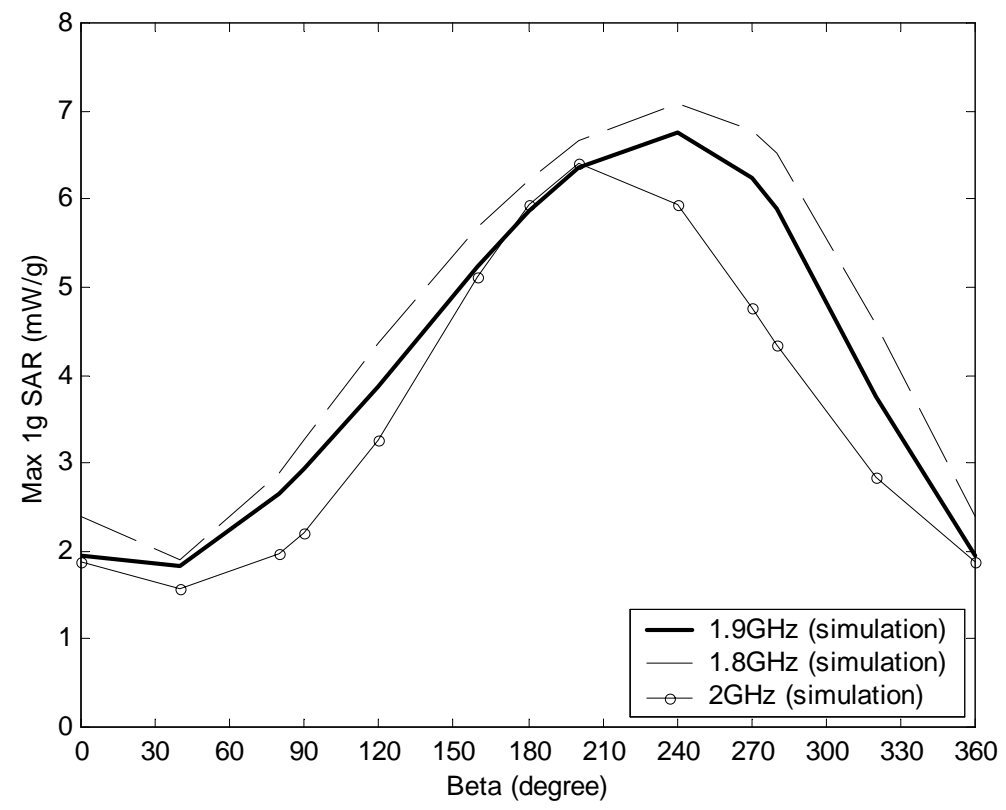

Figure 8. Simulation of maximum $1 \mathrm{~g}$ SAR verses weight phase (Beta) for three frequencies $1.8 \mathrm{G}, 1.9 \mathrm{G}$ and $2 \mathrm{G}$. 